\title{
Fiber-Optic Breath Sensors: A Comparison Study
}

\author{
Jan Nedoma ${ }^{1, a}$, Marcel Fajkus ${ }^{1, b}$, Radek Martinek ${ }^{2, c}$ \\ ${ }^{1}$ Department of Telecommunications, Faculty of Electrical Engineering and Computer Science \\ VSB-Technical University of Ostrava, Ostrava, Czech Republic \\ ${ }^{2}$ Department of Cybernetics and Biomedical Engineering, Faculty of Electrical Engineering and \\ Computer Science, VSB-Technical University of Ostrava, Ostrava, Czech Republic
}

ajan.nedoma@vsb.cz, bmarcel.fajkus@vsb.cz, cradek.martinek@vsb.cz,

Keywords: Respiratory rate (RR), fiber-optic sensor, Fiber Bragg grating (FBG).

\begin{abstract}
The paper presents a comparative study of three fiber optic sensors based on the fiber Bragg grating (FBG). The basic monitored parameter is the respiratory rate of the human body. Fiber-optic sensors are immune to electromagnetic interference (EMI). This fact singles them out as ideal for use in magnetic resonance environments (typically in MRI - magnetic resonance imaging) as a prediction of hyperventilation states in patients. These patient conditions arise as a result of the closed tunnel environment in MR scanners. The results (10 volunteers with written consent) were compared with the results using the conventional respiratory belt $(\mathrm{RB})$ in a laboratory environment and processed using the objective Bland-Altman (B-A) method.
\end{abstract}

\section{Introduction}

Hyperventilation is a state associated with breathing activity. However, it is not a respiratory disease. Hyperventilation indicates accelerated breathing often in a quiescent state. As a result, there is a lack of carbon dioxide in the blood, resulting in hypocapnia of the organism. This process triggers hyperventilation tetanic symptoms. These symptoms are mainly tingling, or numbing, both in the limbs and throughout the body, mouth tingling and feelings of lack of air. Gradually, hyperventilative tetanus passes into strong convulsions throughout the body and unintentional jerky movements. The patient may be disoriented and irritable. Hyperventilation tetanus may last for minutes or even hours. The most common trigger is stressful situations (typically the MR scanner's tunnel environment), which causes an anxiety alarm reaction of the organism. [1-2]

The basic method of predicting hyperventilation states in MR testing today is the use of costly devices. These devices monitor breathing rate, pulse rate or both parameters at the same time. Their price, however, is very high and their dimensions large. Heart rate monitoring typically uses a special ECG (electrocardiography). The basis is 3-lead, but today, the most commonly used are 12 lead ECGs. Special electrodes are used, and the signal is then transmitted wirelessly to the control room [3-4].

Today, in clinical practice, pneumatic or piezoelectric respiration belts are used to monitor breathing frequency [5]. They are placed as needed on the thoracic or abdominal part of the body. These are relatively high-precision devices yet compared to fiber-optic sensors (the price of our sensors is approximately 80 dollars / sensor), their price is relatively high.

Fiber-optic sensors here represent a suitable alternative (they can compete with the price, dimensions and accuracy of sensing vital functions of the human body). Their proliferation is attested to, for example, by publications [6-11]. This article introduces 3 different FBG sensors that differ in their form of encapsulation. These sensors have been presented individually by our author collective in previous research (chest-placed PDMS sensor [12], the fiberglass sensor (fiberglass is a composite material made up of glass fiber (fabric) and cured synthetic resin [13]) and PDMS PAD placed on the back [14]). 
This publication presents the original results by comparing these sensors simultaneously among each other in contrast to the conventional, clinically-used respiratory belt (RB) during monitoring the breathing frequency of the human body.

\section{Methods}

The method of measuring the breathing frequency in the case of the three sensors mentioned above, uses the encapsulation of the Fiber Bragg Grating (FBG). The Bragg grating consists of a periodic structure of changes in the refraction index in the core of the optical fiber, please see Figure 1.

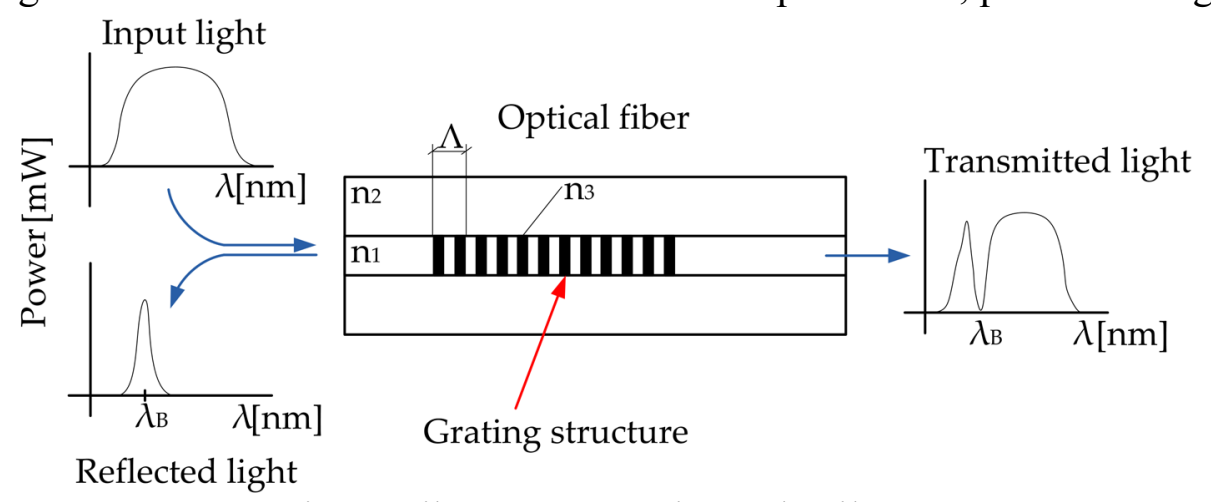

Fig. 1. Fiber Bragg grating - detail.

If the Bragg grating is appropriately encapsulated, a sensor is created that can be placed on a person's chest. The breath rate monitor uses body movements or the chest basket expansion, which is caused by inhalation and exhalation. As a result of this expansion of the thorax, pressure is exerted on the Bragg's grating, that is, changes occur in the geometrical and optical properties of the FBG sensor. These changes are manifested by the shifting of the spectral region of reflected light, referred to as the Bragg wavelength.

$$
\lambda_{B}=2 n_{e f f} \Lambda,
$$

where $\lambda_{B}$ je is the Bragg wavelength, $n_{e f f}$ is the effective refractive index and $\Lambda$ is the spatial period of grating structure [15]. The overall quantity of the proposed FBG probes can be given by:

$$
\frac{\Delta \lambda_{B}}{\lambda_{B}}=k \varepsilon+\left(\alpha_{\Lambda}+\alpha_{n}\right) \Delta T
$$

where $k$ is the deformation coefficient, $\varepsilon$ the acting deformation (in our case deformation caused by breathing activity), $\alpha_{\Lambda}$ is the coefficient of thermal expansion, $\alpha_{n}$ is the thermo-optic coefficient and finally symbol $\Delta T$ represents the temperature changes.

Breathing frequency is processed using the following methodology, please see Figure 2. Unwanted noise (slight movement artefacts, muscle activity) are created by higher frequencies. On the contrary, the effect of temperature is manifested by lower frequencies. For these reasons, a third-order lowpass filter is used to establish the breathing frequency (Butterworth type) with limit frequencies from 0 to $0.5 \mathrm{~Hz}$ [16]. This is followed by the process of normalization and centering of the signal to the zero, median, value and the detection of peaks. Based on the detection of peaks, the Respiratory Rate $(R R)$ can be established as follows:

$$
R R=60 /\left(t_{n}-t_{n-1}\right),
$$

where $t_{n}$ is the time mark of the $n$-th peak and $t_{n-1}$ is the time mark of the previous peak. 
The final step is to smooth the breathing frequency curve. A median filter (size 3 window) is used. The median filter was chosen because it is statistically more resistant to remote observation than a sliding average. [16]

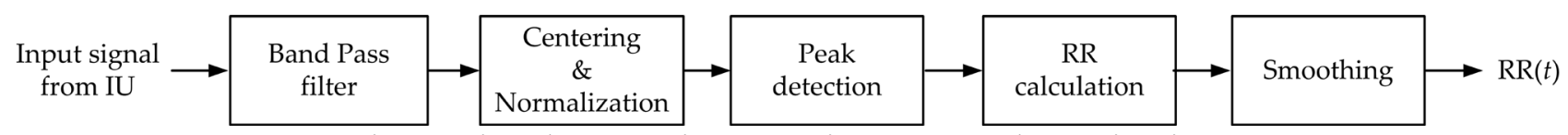

Fig. 2. Signal processing - respiratory rate determination.

Table 1 present baseline characteristics of the study population.

Table 1. Baseline characteristics of the study population.

\begin{tabular}{|c|c|}
\hline & All $(\mathrm{N}=10)$ \\
\hline Age, years & $38 \pm 17$ \\
\hline Females, $\mathrm{n}(\%)$ & $5(50)$ \\
\hline Height, $\mathrm{cm}$ & $169 \pm 14$ \\
\hline Weight, $\mathrm{kg}$ & $68 \pm 14$ \\
\hline BMI, $\mathrm{kg} / \mathrm{m} 2$ & $25.3 \pm 2.5$ \\
\hline Systolic blood pressure, $\mathrm{mmHg}$ & $124 \pm 16$ \\
\hline Diastolic blood pressure, $\mathrm{mmHg}$ & $74 \pm 7$ \\
\hline
\end{tabular}

\section{Experimental setup and results}

The experimental part of this publication is based on evaluated data obtained using 10 healthy volunteers with their written consent. The group of healthy 10 people consists of both males and females ages 21 to 55. The data obtained were analyzed using an objective Bland-Altman method [17]. This method is based on an assessment of the differences between the types of equipment used (our sensors and respiratory reference). The reproducibility is considered good if $95 \%$ of the results lie within a 1.96 SD (Standard Deviation) range. The test subjects were asked to perform the most realistic MR simulation (i.e. during the measurement they were at lying down, and at rest).

A conventional spectral evaluation unit (IU) was used to evaluate the data, please see [18]. The evaluation unit works with a SLED (super luminescent diode) with a power of $1 \mathrm{~mW}$, a central wavelength of $1550 \mathrm{~nm}$, and a sample rating of $1 \mathrm{kHz}$. The pneumatic respiratory belt (RB) was used as a reference, please see [5]. The data was processed according to the manufacturer's instructions.

Figure 3 shows a diagram of experimental measurement. We can see a placement of two FBG sensors (PDMS sensor and Fiberglass sensor) on the chest of the test subject and one FBG sensor (PDMS PAD) on the back of the test subject, also the placement of the reference on the chest (respiratory belt) can be observed. Both FBG chest sensors (PDMS sensor and Fiberglass sensor) were placed under the respiratory belt (our chest sensors are placed beneath the elastic fastening belt, whereas in the case of this study, we used an elastic respiratory reference belt).

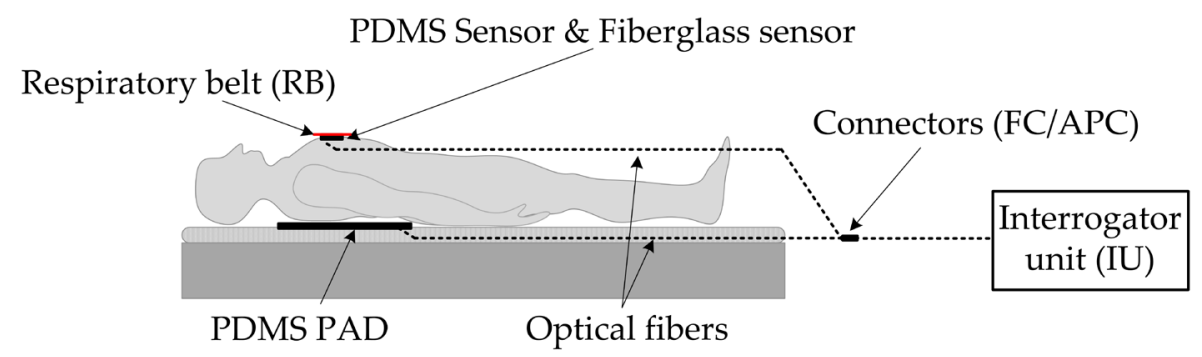

Fig. 3. Experimental scheme.

Figure 4 shows photographs of the three FBG sensors used. These are sensor prototypes that were used within the context of the experimental measurements in this publication. 


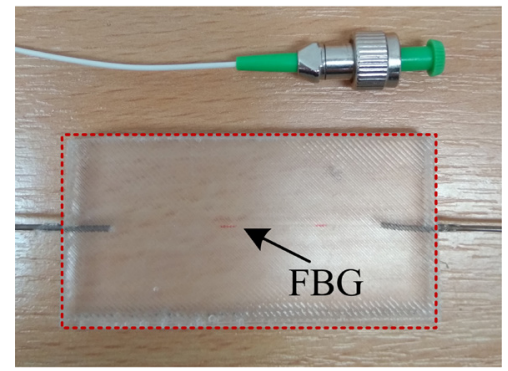

(a)

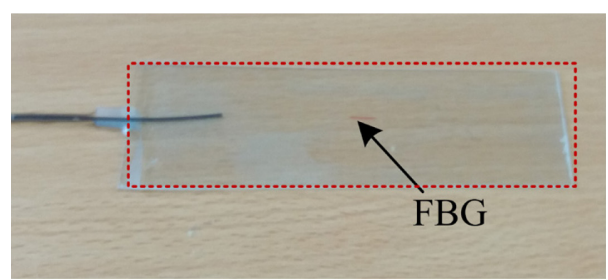

(b)

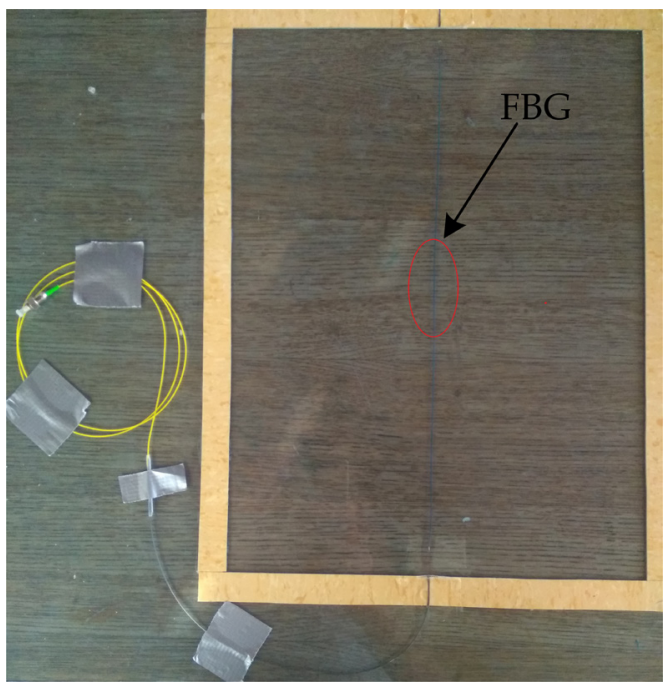

(c)

Fig. 4. A photo of FBG sensors: (a) PDMS sensor (chest); (b) Fiberglass sensor (chest); (c) PDMS PAD.

Figure 5 shows an example of a graphical comparison of the above-mentioned respiratory signals. The figure 5 shows 10-second details. Figure 5(a) represents the progression (male volunteer 1) obtained by the PDMS sensor (chest) vs. reference (RB), Figure 5(b) represents the progression (male volunteer 2) obtained by the Fiberglass sensor (chest vs. reference (RB), Figure 5(c) represents the progression (female volunteer 1) obtained by PDMS PAD vs. Reference (RB). 


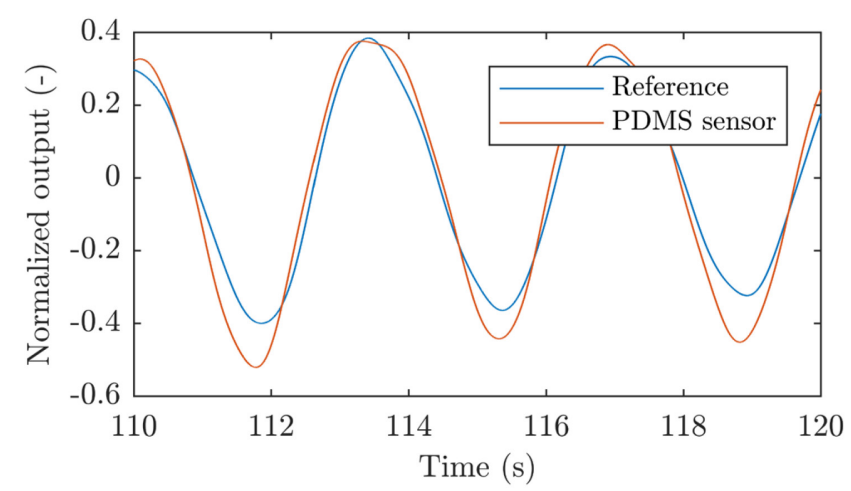

(a)

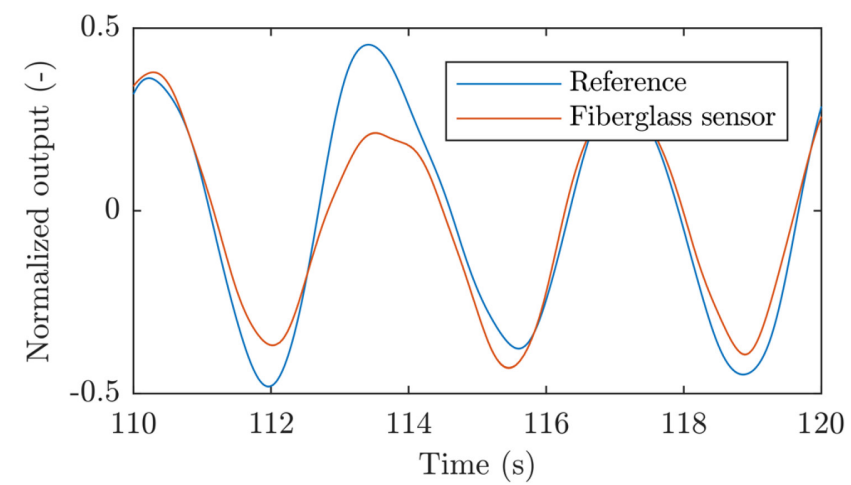

(b)

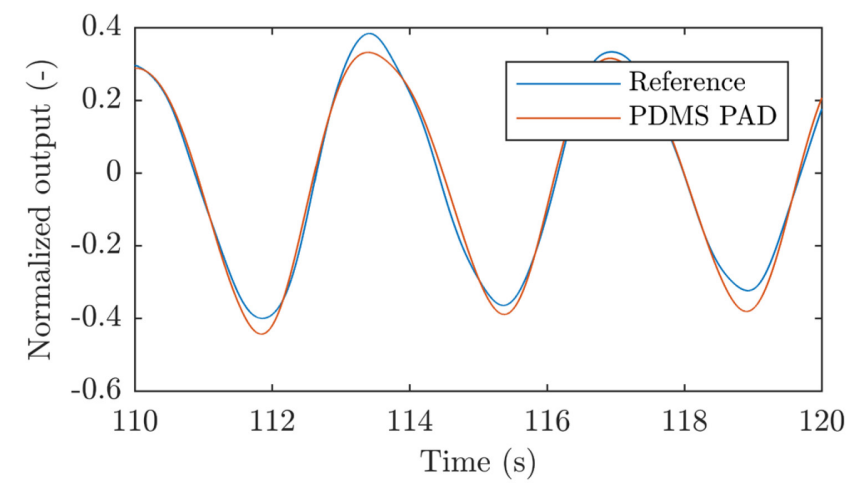

(c)

Fig. 5. A 10-second detail of respiratory activity: (a) PDMS sensor (chest) vs. reference (RB), (b) Fiberglass sensor (chest) vs. reference (RB), (c) PDMS PAD vs. reference (RB).

Figure 6 shows an example of a graphical comparison of the above-mentioned respiratory signals. We displayed for the better clarity 100-second courses of breathing activity which compares individual sensors against the reference respiratory belt. Figure 6(a) represents the progression (male volunteer 1) obtained by the PDMS sensor (chest) vs. reference (RB), Figure 6(b) represents the progression (male volunteer 2) obtained by the Fiberglass sensor (chest vs. reference (RB), Figure 6(c) represents the progression (female volunteer 1) obtained by PDMS PAD vs. Reference (RB). 


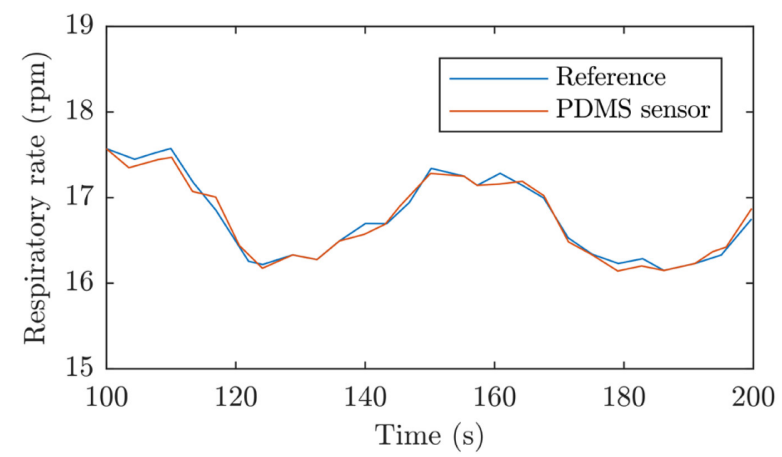

(a)

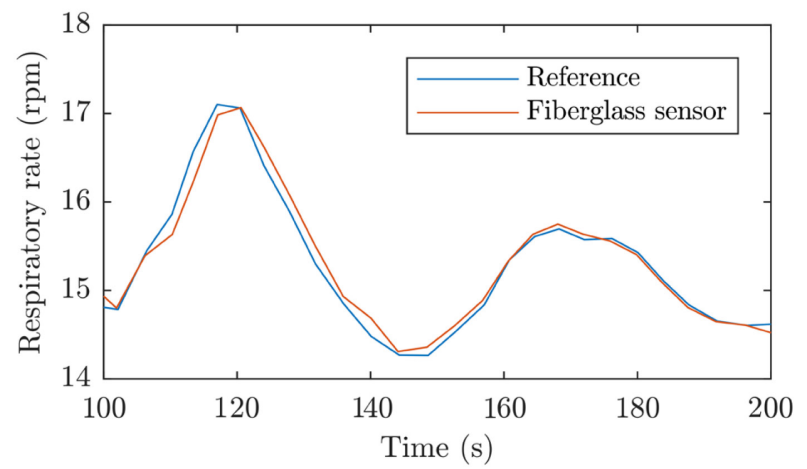

(b)

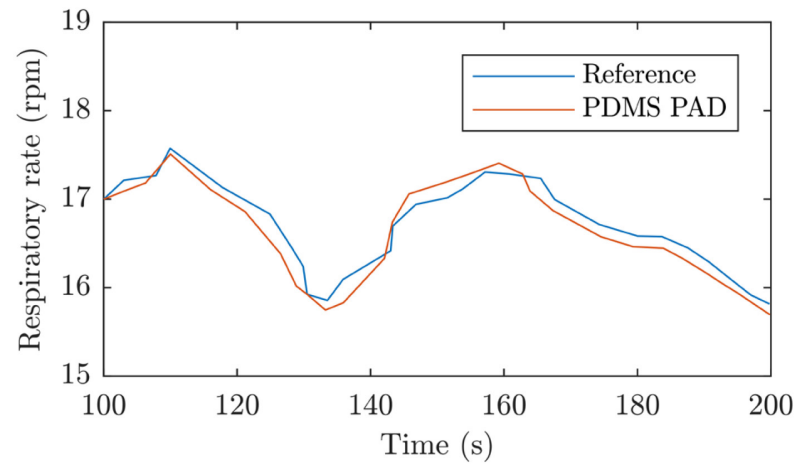

(c)

Fig. 6. A 100-second courses of respiratory activity: (a) PDMS sensor (chest) vs. reference (RB), (b) Fiberglass sensor (chest) vs. reference (RB), (c) PDMS PAD vs. reference (RB).

Table 1 summarizes the results. Total record time of the individual test volunteers is represented as Rec. Time (seconds), further is shown the total number of samples recorded by the FBG sensors (NoS sensor) and the results of the B-A analysis (Samples (\%) in $\pm 1.96 \mathrm{SD}$ ).

Table 1. Summary of results (all three type of sensors).

\begin{tabular}{|c|c|c|c|c|c|c|c|}
\hline \multirow[b]{2}{*}{ Subject } & \multirow[b]{2}{*}{$\begin{array}{l}\text { Rec. time } \\
\text { (s) }\end{array}$} & \multicolumn{2}{|c|}{ RR - PDMS } & \multicolumn{2}{|c|}{ RR - Fiberglass } & \multicolumn{2}{|c|}{ RR - PDMS PAD } \\
\hline & & $\begin{array}{c}\text { NoS } \\
\text { sensor } \\
{[-]}\end{array}$ & $\begin{array}{c}\text { Samples in } \\
\pm 1.96 \mathrm{SD} \\
(\%)\end{array}$ & $\begin{array}{c}\text { NoS } \\
\text { sensor } \\
{[-]}\end{array}$ & $\begin{array}{c}\text { Samples in } \\
\pm 1.96 \mathrm{SD} \\
(\%)\end{array}$ & $\begin{array}{c}\mathrm{NoS} \\
\text { sensor } \\
{[-]}\end{array}$ & $\begin{array}{c}\text { Samples in } \\
\pm 1.96 \mathrm{SD} \\
(\%)\end{array}$ \\
\hline M1 & 506 & 160 & 98.27 & 158 & 98.16 & 153 & 97.23 \\
\hline M2 & 571 & 149 & 98.41 & 147 & 98.12 & 143 & 97.47 \\
\hline M3 & 539 & 142 & 97.98 & 138 & 97.44 & 136 & 96.87 \\
\hline M4 & 517 & 131 & 97.59 & 129 & 97.42 & 125 & 96.85 \\
\hline M5 & 528 & 152 & 97.43 & 151 & 97.37 & 149 & 97.11 \\
\hline F1 & 547 & 156 & 95.55 & 155 & 95.43 & 153 & 95.12 \\
\hline F2 & 552 & 138 & 96.98 & 135 & 96.44 & 131 & 95.87 \\
\hline F3 & 531 & 136 & 97.41 & 134 & 97.21 & 128 & 96.24 \\
\hline F4 & 569 & 141 & 97.02 & 139 & 96.74 & 132 & 95.74 \\
\hline F5 & 518 & 131 & 96.13 & 124 & 95.23 & 120 & 94.72 \\
\hline Sum & 5378 & - & 97.27 & & 96.95 & & 96.32 \\
\hline
\end{tabular}




\section{Discussion}

Figures 5 and 6 describe examples of how individual fiber-optic sensors "follow" the course obtained from the conventional, clinically-used respiratory belt. It is very clear that there are some minor deviations. This may be caused by minor movements due to measurements (slight movement or trembling of the legs, torso rotation, slight hand movement, head movement, rapid breathing or coughing or snoring). All these minor aspects are taken into account in the results described above regarding the efficiency of all three sensors.

The signals from the FBG sensors generally correspond more in the case of men test subjects than in the case of the female test subjects. This difference is probably caused by the different physiological constitution of the subjects of the groups. In the follow-up research, we are ready to carry out a detailed analysis of the influence of these above-mentioned artifacts.

\section{Conclusion}

This publication presents the original results of a comparative study of the current issue of using fiber-optic technology in biomedical applications. The results include the use of 3 prototypes of FBG sensors encapsulated into silicone polymer polydimethylsiloxane (PDMS) and fiberglass (fiberglass is a composite material made of glass fiber (fabric) and cured synthetic resin). The measured data were compared using an objective Bland-Altman analysis. The results of the Bland-Altman analysis show satisfactory values (for all three prototypes of FBG sensors more than $95 \%$ of the values lie within \pm 1.96 standard deviation range). The most accurate results were recorded for the prototype PDMS sensor (chest), where a value of $97.27 \%$ was recorded. Fiberglass sensor is characterized by the value $96.95 \%$ and PDMS PAD sensor by the value $96.32 \%$.

\section{Acknowledgement}

This article was supported by the Ministry of Education of the Czech Republic (Project Nos. SP2019/85 and SP2019/80). This work was supported by the European Regional Development Fund in the Research Centre of Advanced Mechatronic Systems project, project number CZ.02.1.01/0.0/0.0/16_019/0000867 within the Operational Programme Research, Development and Education.

\section{References}

[1] F. Kovacs, C. Horvat, A.T. Balogh, G. Hosszu, Fetal phonocardiography, Past and future possibilities. 104 (2010) 19-25.

[2] M. Weckesser, S. Posse, U. Olthoff, L. Kemna, S. Dager, H.W. Müller-Gärtner, Functional imaging of the visual cortex with bold-contrast MRI: Hyperventilation decreases signal response, Magn. Reson. Med. 41 (1999) 213-216.

[3] N.D. Giardino, S.D. Friedman, S.R. Dager, Anxiety, Respiration and Cerebral Blood Flow: Implications for Functional Brain Imaging, Compr. Psychiatry. 48 (2007) 103-112.

[4] M. Zabel, B. Acar, T. Klingenheben, M.R. Franz, S.H. Hohnloser, M. Malik, Analysis of 12-lead T-wave morphology for risk stratification after myocardial infarction, Circulation. 102 (2000) 12521257.

[5] R. Ogura, Y. Hiasa, T. Takahashi, K. Yamaguchi, K. Fujiwara, Y. Ohara, S. Hosokawa, Specific findings of the standard 12-lead ECG in patients withtakotsubo'cardiomyopathy, Circ. Journal. 67 (2003) 687-690.

[6] C. Rotariu, C. Cristea, D. Arotaritei, R.G. Bozomitu, A. Pasarica, Continuous respiratory monitoring device for detection of sleep apnea episodes, In: Proceedings of the 2016 IEEE 22nd International Symposium for Design and Technology in Electronic Packaging. (2016) 106-109. 
[7] D. Tosi, M. Olivero, G. Perrone, Low-cost fiber bragg grating vibroacoustic sensor for voice and heartbeat detection, Appl. Opt. 47 (2008) 5123-5129.

[8] J. Wo, H. Wang, Q. Sun, P.P. Shum, D. Liu, Noninvasive respiration movement sensor based on distributed Bragg reflector fiber laser with beat frequency interrogation, Journal Biomed. Opt. 19 (2014).

[9] L. Dziuda, F. Skibniewski, A new approach to ballistocardiographic measurements using fibre Bragg grating-based sensors, Biocybern. Biomed. Eng. 34 (2014) 101-106.

[10] W.B. Spillman, M. Mayer, J. Bennett, J. Gong, K.E. Meissner, B. Davis, X. Xu, A 'smart' bed for non-intrusive monitoring of patient physiological factors, J. Meas. Sci. Technol. 15 (2004) 16141620.

[11] J. Witt, F. Narbonneau, M. Schukar, K. Krebber, J. De Jonckheere, M. Jeanne, T. Thiel, Medical textiles with embedded fiber optic sensors for monitoring of respiratory movement, IEEE Sensors Journal 12 (2012) 246-254.

[12] J. Nedoma, M. Fajkus, P. Siska, R. Martinek, V. Vasinek, Non-invasive fiber optic probe encapsulated into PolyDiMethylSiloxane for measuring respiratory and heart rate of the human body, Adv. Electr. Electr. Eng. 15 (2017) 93-100.

[13] J. Nedoma, M. Fajkus, J. Jargus, R. Martinek, K. Witas, J. Vanus, V. Vasinek, Analysis of encapsulation the fiber Bragg sensors for biomedical applications, In: Proceedings of SPIE - The International Society for Optical Engineering. 10654 (2018).

[14] J. Nedoma, M. Fajkus, J. Cubik, S. Kepak, R. Martinek, J. Vanus, R. Jaros, SMART medical polydimethylsiloxane for monitoring vital signs of the human body, In: IEEE International Conference on E-health Networking, Application \& Services. (2018).

[15] M. Fajkus, J. Nedoma, R. Martinek, V. Vasinek, H. Nazeran, P. Siska, A Non-invasive Multichannel Hybrid Fiber-optic Sensor System for Vital Sign Monitoring, Sensors. 17 (2017) 111.

[16] J. Nedoma, S. Kepak, M. Fajkus, J. Cubik, P. Siska, R. Martinek, P. Krupa, Magnetic Resonance Imaging Compatible Non-Invasive Fibre-Optic Sensors Based on the Bragg Gratings and Interferometers in the Application of Monitoring Heart and Respiration Rate of the Human Body: A Comparative Study, Sensors. 18 (2018) 3713.

[17] J.M. Bland, D.G. Altman, Measuring agreement in method comparison studies, Stat. Methods Med. Res. 8 (1999) 135-160.

[18] Products. Available online: http://www.safibra.cz/en/fbguard-interrogation-unit [2018-11-20]. 